\title{
Constraints on Quintessence Using Recent Cosmological Data
}

\author{
L P L Colombo and M Gervasi \\ Dipartimento di Fisica "G Occhialini", Università di Milano-Bicocca, Piazza della \\ Scienza, 3 I20126 Milano, Italy and INFN Sezione di Milano-Bicocca \\ E-mail: loris.colombo@mib.infn.it
}

\begin{abstract}
Recent data, including the three-year WMAP data, the full $2 \mathrm{dF}$ galaxy power spectrum and the first-year data of the Supernova Legacy Survey, are used to constrain model parameters in quintessence cosmologies. In particular, we discuss the inverse power-law (RP) and SUGRA potentials and compare parameter constraints with those for $\Lambda$ CDM. Both potentials fit current observations with a goodness of fit comparable or better than $\Lambda \mathrm{CDM}$. The constraints on the energy scale $\Lambda_{\mathrm{DE}}$ appearing in both potential expressions are however different. For RP, only energy scales around the cosmological constant limit are allowed, making the allowed models quite similar to $\Lambda$ CDM. For SUGRA, $\Lambda_{\mathrm{DE}}$ values approximately up to Electroweak energy scale are still allowed, while other parameter intervals are slightly but significantly displaced. In particular a value of the primeval spectral index $n_{s}=1$ is still allowed at the $95 \%$ c.l., and this can have an impact on constraints on possible inflationary potentials.
\end{abstract}

Submitted to: JCAP 


\section{Introduction}

In the last two decades, observations of high redshift supernovae [1] showed that the expansion of the Universe is undergoing a phase of positive acceleration. Together with measurements of Large Scale Structure [2] and of anisotropies of the Cosmic Microwave Background (CMB) temperature and polarization [3], these data can be explained if about $70 \%$ of the energy density of our universe is made up by a component with negative pressure, dubbed Dark Energy (DE).

The basic candidate for DE is the cosmological constant, $\Lambda$, with an equation of state $w \equiv p / \rho=-1$. As showed by the three-year Wilkinson Microwave Anisotropy Probe (WMAP) observations 4], a power-law $\Lambda$ CDM model with adiabatic initial conditions is a good fit to most cosmological data [5]. Within the framework of a $\Lambda$ CDM cosmology, the combination of the three-year WMAP (WMAP3) observations with LSS and SNIa data, allowed to measure several fundamental cosmological parameters with percent accuracy. However, a cosmological constant has severe problems on theoretical grounds, in particular fine-tuning and cosmic coincidence.

Several alternatives to a cosmological constants have been proposed, including a perfect fluid with constant $w \neq-1$; self-interacting scalar fields, with a time depending $w>-1$ (quintessence [6, 7]) or $w<-1$ (phantom [8]), or with non-canonical kinetic term (k-essence [9]; quintom [10]); modifications of General Relativity 11].

Quintessence models are particularly interesting due to the existence of tracker potentials. Under the proper assumptions for the potential [12], the energy density of quintessence follows closely the evolution of the dominant component, though decreasing more slowly. In addition, the scalar field evolution does not depend on the initial conditions, lessening fine-tuning and related problems. On the contrary, dynamics depend on the current energy density of DE and on the shape of the scalar field potential $V(\phi)$ and its parameters, which should be added to the usual cosmological ones during data analysis.

Several authors used a combination of WMAP3 data and other cosmological data, to study DE properties. The WMAP team placed constraints on the value of $w$, assumed constant, while other works studied the evolution of $\rho_{\mathrm{DE}}$ [13, or assumed phenomenological parameterization for $w(a)$ [14. However, the resulting constraints may depend on the choice of parameterization (e.g. [15]) and, in general, do not translate directly into constraints on the shape of the potential.

In this work we use recent cosmological data to directly constrain the shape of two classes of quintessence potentials: the Ratra-Peebles Potential (RP [16]) and the SUGRA potential [7. The plane of the paper is as follow. In Sec. 2 we outline the models studied together with the data and the method used. In Sec. 3 we describe our results and discuss them in Sec. 4. 


\section{Models and Data}

In the limit of spatial homogeneity, the equation of motion for a quintessence scalar field $\phi$ is given by

$$
\ddot{\phi}+2 H \dot{\phi}+a^{2} V^{\prime}(\phi)=0
$$

where dots denote derivatives with respect to conformal time $\tau$, the Hubble parameter $H \equiv \dot{a} / a$, and we used natural units, $c=\hbar=1$. The energy and pressure associated with the field are given by

$$
\rho_{\phi}=\frac{1}{2}\left(\frac{\dot{\phi}}{a}\right)^{2}+V(\phi) ; \quad p_{\phi}=\frac{1}{2}\left(\frac{\dot{\phi}}{a}\right)^{2}-V(\phi) .
$$

Together with Friedman equations, (11) and (2) allow to solve for the background evolution in a quintessence cosmology, once the the potential $V(\phi)$, and the density parameters of the different components $\Omega_{\mathrm{b}}, \Omega_{\mathrm{c}}$, etc., are assigned. At variance with a cosmological constant, quintessence is not a smooth component and perturbations in the scalar field need to be accounted for [17]. In the Synchronous gauge they obey the equation

$$
\ddot{\varphi}+2 H \dot{\varphi}+a^{2} V^{\prime \prime}(\phi)+\frac{1}{2} \dot{\mathfrak{h}} \dot{\phi}+\nabla^{2} \varphi=0,
$$

where $\varphi \equiv \delta \phi$ and $\mathfrak{h}$ is the trace part of the metric perturbation. Perturbation evolution is also fixed by the potential.

Quintessence cosmologies, then, clearly depend on shape of $V(\phi)$, which in principle is totally arbitrary. Considering only tracker potentials allow to ease the fine tuning problem of $\Lambda \mathrm{CDM}$ cosmologies. As said in Sec. 1, in this work we focus on two classes of tracker potentials, RP and SUGRA:

$$
\begin{array}{rlrl}
V(\phi) & =\frac{\Lambda_{\mathrm{DE}}^{4+\alpha}}{\phi^{\alpha}}, & \mathrm{RP} \\
V(\phi) & =\frac{\Lambda_{\mathrm{DE}}^{4+\alpha}}{\phi^{\alpha}} \exp \left(\frac{4 \pi \phi^{2}}{m_{p}^{2}}\right), & & \text { SUGRA }
\end{array}
$$

here $m_{p}$ is the Planck mass. The former was one of the first tracker potentials studied, and is characterized by a slowly varying equation of state. The latter originates within the context of Supergravity theories and the corresponding equation of state undergoes a rapid transition when the field becomes dominant. Both potentials depend on two parameters, the slope $\alpha$ and the energy scale $\Lambda_{\mathrm{DE}}$. For a given choice of parameters, the scalar field associated with the two potentials evolve in a similar way until the epoch of DE domination; at that point in a SUGRA model $w(a)$ rapidly falls toward values $-1 \lesssim w(1) \lesssim-0.9$, while in a RP model $w(a)$ decreases more slowly, and stays at considerably higher values.

In a cosmological context, $\alpha$ and $\Lambda_{\mathrm{DE}}$ are not independent; fixing the current energy density of DE, $\rho_{\mathrm{DE}, 0}$, leaves only one free parameter. Notice that for $\alpha \simeq 0$, we recover the behaviour of the cosmological constant. Here, we take $\Lambda_{\mathrm{DE}}$ as a free parameter; however, different conventions are possible [18, 19]. 
In this work, we test RP and SUGRA models using recent data, and try to place constraints on the value of $\Lambda_{\mathrm{DE}}$. We assume a power-law spectrum of primordial density fluctuations with adiabatic initial conditions, and restrict our analysis to flat cosmologies with no massive neutrinos, so that $\Omega_{\mathrm{DE}}=1-\Omega_{\mathrm{b}}-\Omega_{\mathrm{c}}$. The models studied are thus specified by seven parameters: the physical baryon, $\omega_{\mathrm{b}} \equiv \Omega_{\mathrm{b}} h^{2}$, and $\mathrm{CDM}, \omega_{c}$, densities; the angular size of the sound horizon at recombination $\theta_{s}$; the slope, $n_{s}$, and amplitude, $A_{s}$, of the power-spectrum of density fluctuations; the optical depth to reionization, $\tau$; and $\lambda \equiv \log _{10}\left(\Lambda_{\mathrm{DE}} / \mathrm{GeV}\right)$. Notice that $h$ is the reduced Hubble parameter, $H_{0}=100 h \mathrm{Km} / \mathrm{s} / \mathrm{Mpc}$. This choice of parameters is particularly efficient in minimizing the impact of the degeneracies of CMB angular power spectra on data analysis [20]. To explore the parameter space, we used a modified version of the COSMOMC package. We assumed a flat priors on all parameters; in particular $-11.75 \lesssim \lambda \lesssim 19$. The upper limit corresponds to the Planck mass, while the lower one is set by $\left(\Lambda_{\mathrm{DE}}\right)^{4} \simeq 10^{-47} \mathrm{GeV}^{4}$, i.e. the cosmological constant energy density in a concordance $\Lambda$ CDM model. We also tested that a different choice of priors, in particular using $H_{0}$ instead of $\theta_{s}$, does not significantly alter our results.

Models were tested against WMAP3 data, the galaxy power spectrum $P(k)$ of the full 2dF Galaxy Redshift Survey 21] and the distance measurements to high-redshift supernovae by the Supernova Legacy Survey (SNSL 22]). Assuming the three sets of data to be independent, the total likelihood is the product of the individual likelihoods

$$
\mathcal{L} \text { tot }=\mathcal{L}_{\mathrm{CMB}} \times \mathcal{L}_{\mathrm{LSS}} \times \mathcal{L}_{\mathrm{SN}}
$$

We also define an effective $\chi^{2} \equiv-2 \ln (\mathcal{L})$.

In the analysis of the CMB anisotropy spectrum, the WMAP team included the contribution of secondary anisotropies due to Sunayev-Zel'dovich (SZ) effect [5]. The SZ contribution was parametrized in term of the amplitude of the signal, $A_{\mathrm{SZ}}$, relative to a reference $\Lambda \mathrm{CDM}$ model and final constraints were obtained after marginalization over $A_{\mathrm{SZ}}$. The effects of SZ marginalization on $\Lambda \mathrm{CDM}$ estimates are very small. In addition, the SZ contribution depends on the mass function and its evolution; these, in turn, depend on the quintessence model considered and require extensive numerical simulations [23]. Thus, we set $A_{\mathrm{SZ}}=0$ here. We also did not include small scale data in our analysis of CMB anisotropies. High- $\ell$ 's mostly probe the recombination history and the running of the spectral index, but are not significantly affected by the quintessence models we considered.

Analysis of LSS data requires the introduction of an additional parameter, $b$, accounting for the bias in the clustering of galaxies with respect to the clustering of the matter field. Here, we assume that $b$ is independent of scale in the range of $0.02 h \mathrm{Mpc}^{-1}<k<0.15 \mathrm{~h} \mathrm{Mpc}^{-1}$ considered [21], and the code performs an analytic marginalization over it. In these range of wavenumbers non-linear corrections are small [21], and we do not include them in the analysis. For both SUGRA and RP potentials we run 10 chains of $\sim 50000$ points each. We also run a set of chains for a $\Lambda \mathrm{CDM}$ model. Comparing results for quintessence with those for $\Lambda \mathrm{CDM}$ allows then to 
Table 1. SUGRA parameters. For all parameters except $\Lambda_{\mathrm{DE}}$ we show the mean value $\langle x\rangle=\int d x^{\prime} \mathcal{L}\left(x^{\prime}\right) x^{\prime}$ and corresponding $68 \%$ confidence level interval, for the different combinations of datasets used. For $\Lambda_{\mathrm{DE}}$ we show the $95 \%$ upper confidence limit.

\begin{tabular}{ccccc}
\hline Parameter & WMAP3 & WMAP3 + 2dF & WMAP3 + SNLS & $\begin{array}{c}\text { WMAP3 + 2dF } \\
+ \text { SNLS }\end{array}$ \\
\hline $100 \Omega_{b} h^{2}$ & $2.252_{-0.078}^{+0.077}$ & $2.246_{-0.078}^{+0.078}$ & $2.234_{-0.079}^{+0.074}$ & $2.242_{-0.077}^{+0.073}$ \\
$\Omega_{c} h^{2}$ & $0.1032_{-0.0082}^{+0.0082}$ & $0.0980_{-0.0074}^{+0.0072}$ & $0.1001_{-0.0075}^{+0.0071}$ & $0.0991_{-0.0069}^{+0.0069}$ \\
$H_{0}$ & $63.0_{-5.8}^{+6.2}$ & $67.6_{-3.3}^{+3.4}$ & $70.6_{-2.5}^{+2.3}$ & $70.1_{-2.1}^{+2.1}$ \\
$\tau$ & $0.093_{-0.014}^{+0.014}$ & $0.098_{-0.015}^{+0.015}$ & $0.097_{-0.015}^{+0.015}$ & $0.098_{-0.015}^{+0.015}$ \\
$n_{s}$ & $0.967_{-0.020}^{+0.020}$ & $0.965_{-0.019}^{+0.019}$ & $0.961_{-0.018}^{+0.018}$ & $0.963_{-0.018}^{+0.017}$ \\
$\log _{10}\left(\Lambda_{\mathrm{DE}}\right)$ & $<14.9$ & $<9.3$ & $<0.1$ & $<2.1$ \\
$\Omega_{\mathrm{m}}$ & $0.325_{-0.069}^{+0.070}$ & $0.266_{-0.026}^{+0.027}$ & $0.247_{-0.024}^{+0.024}$ & $0.248_{-0.018}^{+0.017}$ \\
$\sigma_{8}$ & $0.634_{-0.086}^{+0.085}$ & $0.648_{-0.082}^{+0.079}$ & $0.696_{-0.063}^{+0.065}$ & $0.685_{-0.069}^{+0.066}$ \\
\hline
\end{tabular}

factor out possible biases introduced by our choice of datasets, priors and methods.

\section{Results}

Table 1 and table 2 summarizes our main results for SUGRA and RP cosmologies, respectively. For each parameter $x$, except $\lambda$, we list the mean value

$$
\langle x\rangle=\int d x^{\prime} \mathcal{L}\left(x^{\prime}\right) x^{\prime}
$$

and the limits of the $68 \%$ confidence interval, defined as appropriate quantiles of the marginalized distribution, for different combinations of data sets. For $\lambda$ we list the upper 95\% confidence limit. Table [3, instead, shows results for a basic 6-parameters $\Lambda \mathrm{CDM}$ cosmology, in order to outline the effects of quintessence.

In figure 1 and figure 3, we plot the marginalized 1D likelihoods, for some basic and derived parameters. Different colours and lines types refer to different combinations of data (see caption for details). In figure 2 and figure 4 we show the joint 2D confidence limits between $\Lambda_{\mathrm{DE}}$ and other relevant parameters.

\section{Discussion}

Using the first-year WMAP (WMAP1) data, several authors 18 placed constraints on the slope of RP potential, finding $\alpha \lesssim 2$. This constraint does not directly translate into a constraint on the energy scale $\Lambda_{\mathrm{DE}}$, as the $\alpha-\Lambda_{\mathrm{DE}}$ relation depends on $\rho_{\mathrm{DE}, 0}$. However, it was clear that allowed values fall well below the $1-10^{6} \mathrm{GeV}$ range, which includes the Electroweak (EW) and (possibly) the Supersymmetry (SUSY) breaking scales. Let us outline soon that WMAP3 data do not improve this situation. While a SUGRA potential still provides a fair fit of data for a physically relevant range of $\Lambda_{\mathrm{DE}}$ values, 

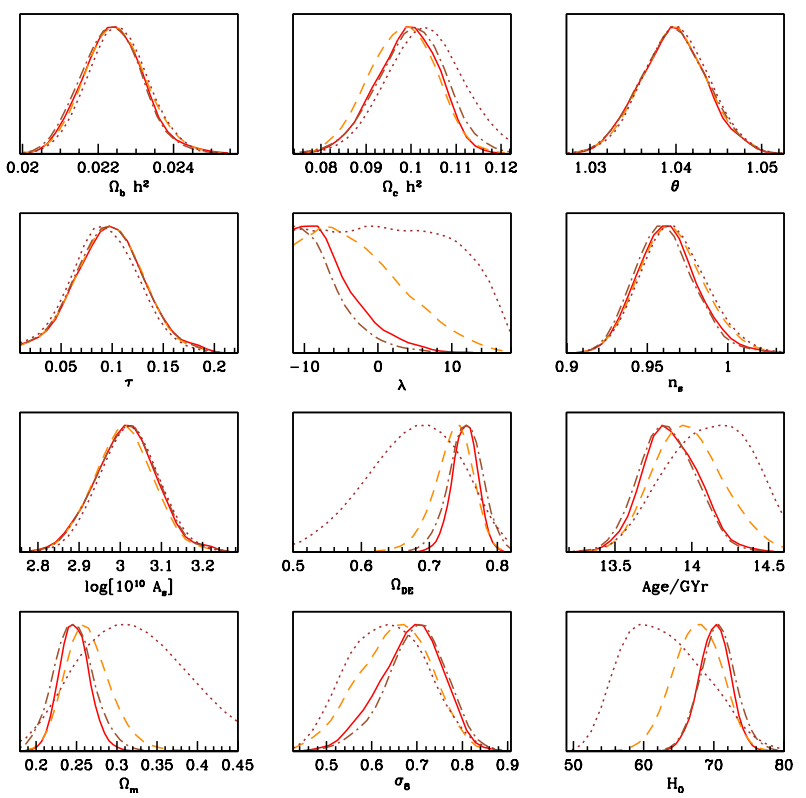

Figure 1. Marginalized 1D constraints on basic and derived parameters of SUGRA cosmology. Dotted lines show constrains from WMAP3 only, dashed lines from WMAP3 + 2dF, dot-dashed from WMAP3 + SNLS and solid lines from the combination of all data. WMAP3-only constraints are very loose due to degeneracies between $\lambda$ and other parameters, especially $\Omega_{\mathrm{m}}$.
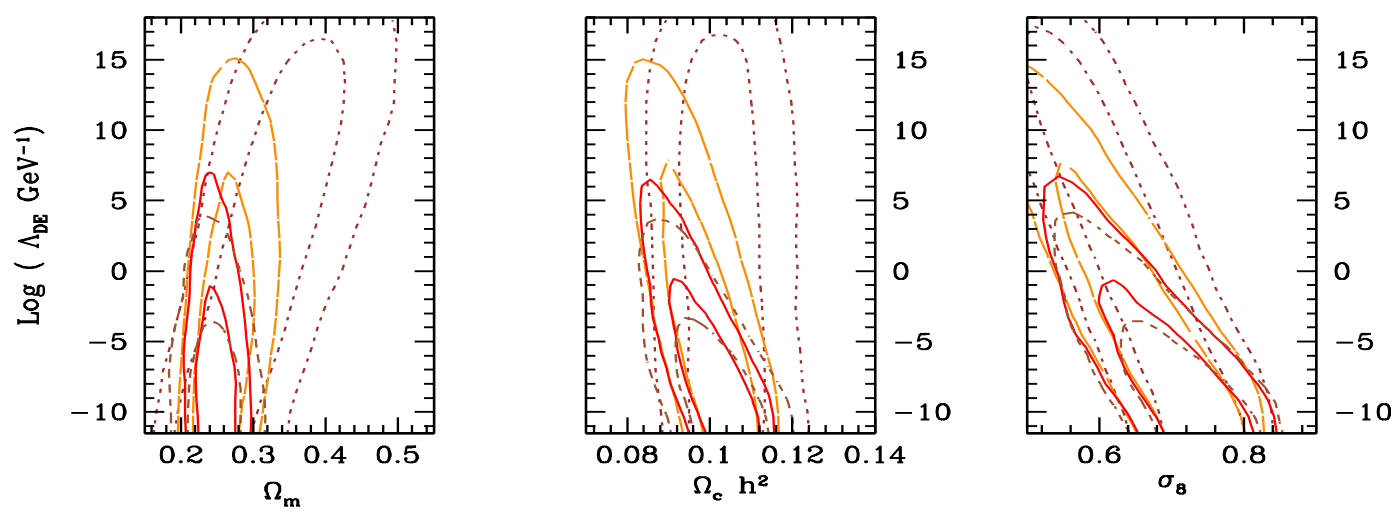

Figure 2. Left: Joint $2 \mathrm{D}$ constraints in the $H_{0}-\lambda$ plane for a SUGRA potential. Lines references are the same as figure 1 Notice the strong degeneracy between the two parameters when using only CMB data. Marginalization over $\lambda$ yields a high value of $\Omega_{\mathrm{m}}$ with a large uncertainty. Adding additional data strongly suppress the high value of $\lambda$ and marginalized constraints are similar to results for $\Lambda$ CDM. Middle: Joint constraints in the $\omega_{c}-\lambda$ plane. Right: Joint constraints in the $\sigma_{8}-\lambda$ plane. 
Constraints on Quintessence Using Recent Cosmological Data

Table 2. RP parameters.

\begin{tabular}{ccccc}
\hline Parameter & WMAP3 & WMAP3 + 2dF & WMAP3 + SNLS & $\begin{array}{c}\text { WMAP3 + 2dF } \\
+ \text { SNLS }\end{array}$ \\
\hline $100 \Omega_{b} h^{2}$ & $2.262_{-0.083}^{+0.083}$ & $2.232_{-0.074}^{+0.072}$ & $2.225_{-0.070}^{+0.074}$ & $2.225_{-0.071}^{+0.070}$ \\
$\Omega_{c} h^{2}$ & $0.1025_{-0.0083}^{+0.0083}$ & $0.0999_{-0.0065}^{+0.0066}$ & $0.1015_{-0.0067}^{+0.0067}$ & $0.1016_{-0.0057}^{+0.0057}$ \\
$H_{0}$ & $59.7_{-8.7}^{+8.7}$ & $68.1_{-3.2}^{+3.3}$ & $71.3_{-2.3}^{+2.3}$ & $70.8_{-1.9}^{+1.9}$ \\
$\tau$ & $0.091_{-0.014}^{+0.014}$ & $0.094_{-0.014}^{+0.014}$ & $0.094_{-0.014}^{+0.014}$ & $0.092_{-0.015}^{+0.014}$ \\
$n_{s}$ & $0.969_{-0.023}^{+0.022}$ & $0.960_{-0.017}^{+0.017}$ & $0.958_{-0.016}^{+0.017}$ & $0.957_{-0.016}^{+0.016}$ \\
$\log _{10}\left(\Lambda_{\mathrm{DE}}\right)$ & $<8.9$ & $<-3.6$ & $<-7.9$ & $<-7.7$ \\
$\Omega_{\mathrm{m}}$ & $0.369_{-0.103}^{+0.109}$ & $0.266_{-0.027}^{+0.025}$ & $0.245_{-0.024}^{+0.025}$ & $0.247_{-0.018}^{+0.019}$ \\
$\sigma_{8}$ & $0.600_{-0.086}^{+0.080}$ & $0.676_{-0.063}^{+0.066}$ & $0.712_{-0.053}^{+0.057}$ & $0.713_{-0.050}^{+0.050}$ \\
\hline
\end{tabular}

Table 3. $\Lambda$ CDM parameters.

\begin{tabular}{ccccc}
\hline Parameter & WMAP3 & WMAP3 + 2dF & WMAP3 + SNLS & $\begin{array}{c}\text { WMAP3 + 2dF } \\
+ \text { SNLS }\end{array}$ \\
\hline $100 \Omega_{b} h^{2}$ & $2.220_{-0.072}^{+0.073}$ & $2.220_{-0.070}^{+0.072}$ & $2.223_{-0.072}^{+0.072}$ & $2.219_{-0.070}^{+0.071}$ \\
$\Omega_{c} h^{2}$ & $0.1054_{-0.0080}^{+0.0079}$ & $0.1070_{-0.0050}^{+0.0050}$ & $0.1075_{-0.0058}^{+0.0058}$ & $0.1077_{-0.0044}^{+0.0044}$ \\
$H_{0}$ & $73.0_{-3.2}^{+3.2}$ & $72.2_{-2.1}^{+2.0}$ & $72.2_{-2.3}^{+2.3}$ & $71.9_{-1.8}^{+1.8}$ \\
$\tau$ & $0.090_{-0.014}^{+0.013}$ & $0.086_{-0.013}^{+0.013}$ & $0.087_{-0.013}^{+0.013}$ & $0.085_{-0.012}^{+0.013}$ \\
$n_{s}$ & $0.955_{-0.016}^{+0.016}$ & $0.953_{-0.016}^{+0.016}$ & $0.953_{-0.016}^{+0.016}$ & $0.953_{-0.016}^{+0.015}$ \\
$\Omega_{\mathrm{m}}$ & $0.242_{-0.035}^{+0.034}$ & $0.248_{-0.022}^{+0.022}$ & $0.250_{-0.025}^{+0.025}$ & $0.252_{-0.018}^{+0.018}$ \\
$\sigma_{8}$ & $0.758_{-0.048}^{+0.048}$ & $0.767_{-0.037}^{+0.037}$ & $0.770_{-0.041}^{+0.041}$ & $0.770_{-0.036}^{+0.036}$ \\
\hline
\end{tabular}

this is not so for RP. We therefore confirm that SUGRA is still a viable cosmology; on the contrary, while RP is compatible with data, the cosmology it yields has just minor differences from $\Lambda$ CDM. Let us then discuss first the SUGRA case, which leads to relevant shifts of some parameters in respect to $\Lambda$ CDM.

\subsection{SUGRA potential}

A first point to be outlined is that there is no appreciable difference between the likelihood of SUGRA and $\Lambda$ CDM, as the $\chi^{2}$ value, for SUGRA, is only very marginally improved.

When only WMAP3 data are considered, the range of allowed $\Lambda_{D E}$ values is essentially unconstrained: $\lambda=\log _{10}\left(\Lambda_{D E} / \mathrm{GeV}\right) \lesssim 15$, at the $95 \%$ confidence level. Considering the whole set of data, the limit is significantly more stringent: $\lambda \lesssim 2.1$ at $95 \%$ c.l. Assuming for the other parameters their expectation values, $\lambda=2.1$ corresponds to $\alpha=3.36$. The strong difference from RP is due to the fact that, at late times, when $\phi$ approaches the Planck mass, the exponential term dominates the 

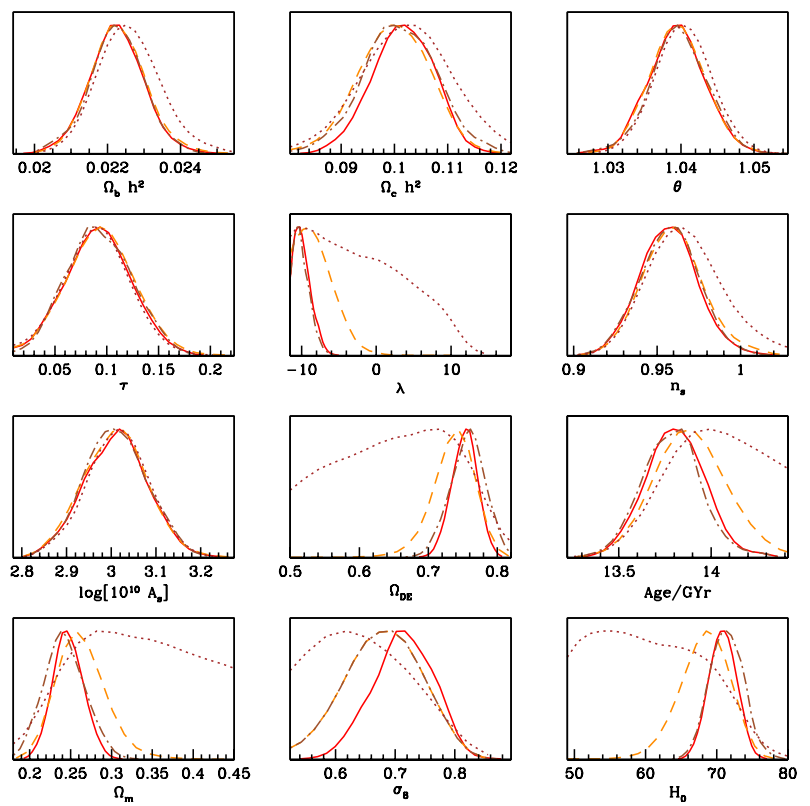

Figure 3. Marginalized $1 \mathrm{D}$ constraints on basic and derived parameters of RP cosmology, for the same combinations of data sets as in figure 1.
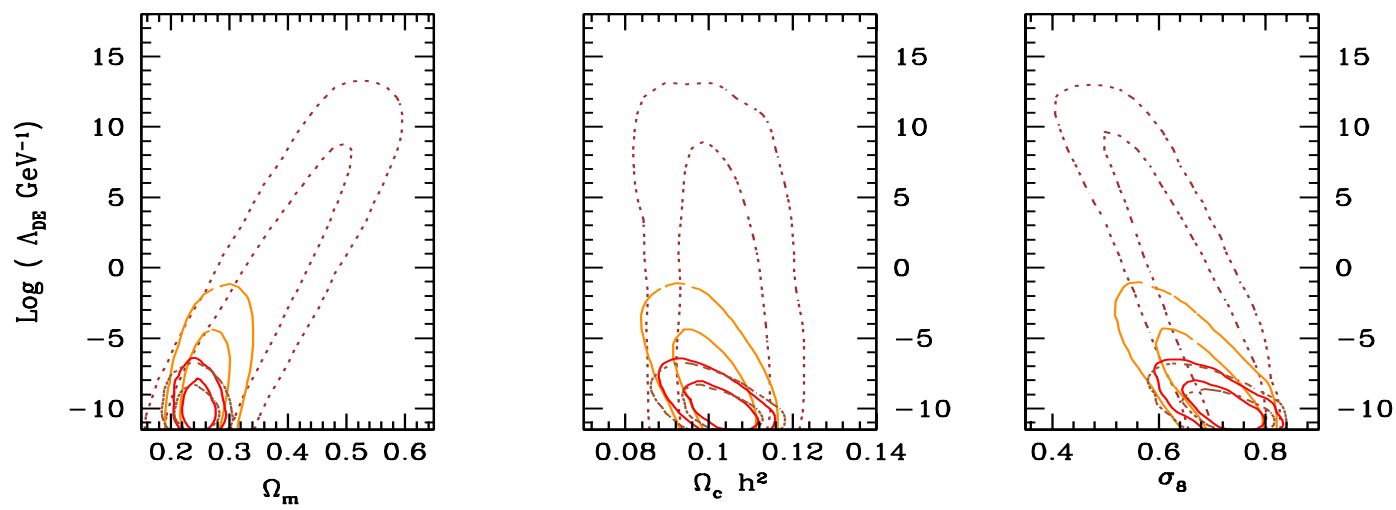

Figure 4. Left: Joint $2 \mathrm{D}$ constraints in the $\Omega_{\mathrm{m}}-\lambda$ plane for a RP potential. Lines references are the same as figure 1

potential, smoothing the dependence on the energy scale.

As far as other parameters are concerned, mean values and confidence intervals are different from $\Lambda \mathrm{CDM}$; shifts are more relevant for $n_{s}, \Omega_{\mathrm{m}}$ and $H_{0}$. The shift of the primeval spectral index $n_{s}$, in particular, affects the impact of WMAP3 data on the nature of inflation [5, 24]. Using the whole data set, we find for $\Lambda$ CDM:

$$
n_{s}=0.953_{-0.016-0.030}^{+0.015+0.031},
$$

while for SUGRA:

$$
n_{s}=0.963_{-0.018-0.032}^{+0.017+0.038} .
$$

Here we have quoted the mean, the $68 \%$ and $95 \%$ c.l. errors; the $95 \%$ errors include the contribution from the $68 \%$ uncertainty. These figures show that a Zel'dovich 
spectrum, excluded at the $95 \%$ c.l. $\left(n_{s}<0.984\right)$, if $\Lambda$ CDM models are considered, is compatible with the whole data set $\left(n_{s}<1.001\right)$, at the same confidence level, for SUGRA cosmologies (see also [25]).

More in detail, let us outline that, when using only WMAP3 data, constraints on $\Omega_{\mathrm{m}}, H_{0}$ and $\sigma_{8}$ are very poor, in qualitative agreement with results for WMAP1 data [26]. In particular, $H_{0}=63 \pm 7 \mathrm{Km} / \mathrm{s} / \mathrm{Mpc}, \Omega_{\mathrm{m}}=0.32 \pm 0.07$ and $\sigma_{8}=0.63 \pm 0.09$. This is due to a strong degeneracy between $\lambda$ and $\Omega_{\mathrm{m}}$, which in turn affects estimates of $H_{0}$ and $\sigma_{8}$, as shown in figure 2. This degeneracy is qualitatively similar to the geometric $w-\Omega_{\mathrm{m}}$ degeneracy in models with a constant equation of state. Fixing all other parameters, the effective value of $\langle w\rangle=\int d a w(a) \Omega_{\mathrm{DE}}(a) / \int d a \Omega_{\mathrm{DE}}(a)$, is an increasing function of $\Lambda_{\mathrm{DE}}$ and the field becomes dominant at earlier times than in $\Lambda$ CDM. When fitting CMB data, this behaviour yields higher $\Omega_{\mathrm{m}}$ and lower $\sigma_{8}$ as $\Lambda_{\mathrm{DE}}$ increases. Notice that there is no one-to-one relation $\Lambda_{\mathrm{DE}^{-}}-w(a)$, and results for models with constant $w$ cannot be straightforwardly applied to constrain the energy scale.

The main difference between WMAP1 and WMAP3 constraints is in the marginalized distribution of $n_{s}$ and $\tau$. The Integrated Sachs-Wolfe effect acts on low$\ell$ anisotropy multipoles more strongly in SUGRA than in $\Lambda$ CDM. In WMAP1, this increases the $\tau-n_{s}$ degeneracy, and both $\tau$ and $n_{s}$ are quite higher for SUGRA than for $\Lambda$ CDM. WMAP3 estimates of TE and EE spectra allows a firm determination of $\tau$, and considering dynamical DE does not significantly worsen constraints on $\tau$ and $n_{s}$.

Including other datasets in the analysis strongly reduces the allowed range for $\Lambda_{\mathrm{DE}}$, in turn significantly affecting the estimates of parameters degenerate with the energy scale. Both LSS and SNIa data exclude the highest values of $\Lambda_{\mathrm{DE}}$, corresponding to models with an high effective $\langle w\rangle$. The combination of WMAP3 and SNLS is particularly constraining for this class of models, and adding also $2 \mathrm{dF}$ data has only minor effects on estimates. In fact, current SN data seem to prefer models with equation of state around or slightly lower than -1 , while for a quintessence model $w(a)>-1$.

SUGRA also favours higher values of $\omega_{\mathrm{b}}$, although differences are around $1 / 3$ of the current uncertainty. As discussed above, $\Lambda_{\mathrm{DE}}$ affects estimates of $\tau$ and $n_{s}$ which in turn affect $\omega_{\mathrm{b}}$ through the $\tau-n_{s}-\omega_{\mathrm{b}}-A_{s}$ degeneracy. However, if additional data allow to establish a clear difference in estimates for SUGRA and $\Lambda$ CDM, Big Bang Nucleosynthesis measures could allow to distinguish the two models.

Let us also outline that SUGRA models show an improvement in the goodness of fit, when LSS data are included in the analysis. Such improvement is modest, $\Delta \chi^{2} \simeq-1$, and does not provide a compelling evidence in favour of SUGRA cosmology. However, the dynamical range of SUGRA models is still very wide and compatible with the energy scales of fundamental physics, and at present they offer a valid alternative to $\Lambda$ CDM.

\subsection{RP potential}

Considering only WMAP3 data, results for RP are qualitatively similar to SUGRA. Upper limits on $\Lambda_{\mathrm{DE}}$ are moderately tighter than in SUGRA, $\lambda \lesssim 8.9$ (95\% c.l.), and 
discrepancies between RP and $\Lambda \mathrm{CDM}$ estimates are greater that those between SUGRA and $\Lambda$ CDM.

However, adding other data strongly suppress the high tail of the distribution on $\Lambda_{\mathrm{DE}}$; using all data, we find $\lambda \lesssim-7.7$ (95\% c.l.), well below the relevant range for EW and SUSY breaking. Moreover, estimates for other parameters are very similar to $\Lambda \mathrm{CDM}$ values, except for $\Omega_{\mathrm{c}} h^{2}$ and $\sigma_{8}$. Both are lower than the corresponding $\Lambda \mathrm{CDM}$ figures by about 1 standard deviation.

As already stated, previous works [18, 19] used various combinations of data to constrain the slope of RP potential, finding that $\alpha \lesssim 2$. A direct comparison of the marginalized distribution on $\alpha$ and $\Lambda_{\mathrm{DE}}$ is not immediate; however, we notice that assuming $\Lambda_{\mathrm{DE}}=10^{-7} \mathrm{GeV}$ we find that $0.7 \lesssim \alpha \lesssim 0.8$ when $\Omega_{\mathrm{DE}}$ varies in the range $0.1-0.9$ and $H_{0}=70 \mathrm{Km} / \mathrm{s} / \mathrm{Mpc}$. For these values of the parameters, the range of initial conditions for which $\phi$ reaches the tracking regime is very small, and RP models have fine tuning problems similar to $\Lambda$ CDM [27]. Choosing initial conditions so that $\phi$ does not reach the tracking regime by the present time, could alter the bounds on the

potential parameters [28]. However, without tracking, quintessence models lose one of their conceptual advantages over other DE candidates.

Thus RP models, while still compatible with present data, do not seem to be a strong alternative to a standard $\Lambda$ CDM cosmology.

\subsection{Summary}

We have used recent cosmological data to constrain the parameters of two relevant quintessence potentials, Ratra-Peebles (RP) and SUGRA. Both potentials depend on an energy scale, $\Lambda_{\mathrm{DE}}$, and the corresponding cosmologies reduce to $\Lambda \mathrm{CDM}$ for $\left(\Lambda_{\mathrm{DE}}\right)^{4} \simeq 10^{-47} \mathrm{GeV}^{4}$.

Both for RP and SUGRA, current data allow to put only upper limits on $\Lambda_{\mathrm{DE}}$, due to a strong degeneracy between $\Lambda_{\mathrm{DE}}$ and $\Omega_{\mathrm{m}}$. When considering only the third-year WMAP data, the degeneracy results in wider errors on several parameters, including $\Omega_{\mathrm{m}}$, $H_{0}$ and $\sigma_{8}$. When LSS and, especially, SN data are added to the analysis, constraints on $\Lambda_{\mathrm{DE}}$ strongly tighten. For RP potential, only values close to the cosmological limit are still allowed, and the model does not offer clear advantages to $\Lambda$ CDM.

For SUGRA potential, instead, values of $\Lambda_{\mathrm{DE}} \simeq 100 \mathrm{GeV}$, close to weak interaction energy scale, are still compatible with data. In addition, estimates of several parameters are different from the corresponding $\Lambda$ CDM values. In particular, the spectral index, $n_{s}$, is compatible with a Zel'dovich spectrum at less than 2 standard deviations. This could have a strong impact on constraints on the shape of the inflation potential. SUGRA models, thus, are still a viable and significant alternative to $\Lambda$ CDM.

\section{Acknowledgments}

We thank Roberto Mainini and Silvio Bonometto for helpful discussions. 
Constraints on Quintessence Using Recent Cosmological Data

\section{References}

[1] Riess A G, Strolger L-G, Tonry J, et al. 2004 Astrophys. J. 607 665; Perlmutter S, Aldering G, Goldhaber G, et al. 1999 Astrophys. J. 517565

[2] Colless M M, Dalton G B, Maddox S J, et al. 2001 Mon. Not. Roy. Astronom. S. 329 1039; Colless M M, Peterson B A, Jackson C, et al. 2003 Preprint astro-ph/0306581; Loveday J (the SDSS collaboration) 2002 Contemporary Phys. 43 437; Tegmark M, Blanton M, Strauss M et al. 2004 Astrophys. J. 606 702-740; Adelman-McCarthy J K, Agueros M A, Allam S S, et al. 2006 Astrophys. J. Suppl. 162 38-48

[3] de Bernardis P, Ade P A R, Bock J J, et al. 2000 Nature 404 955; Padin S, Cartwright J K, Mason B S, et al. 2001 Astrophys. J. 549 L1; Kovac J, Leitch E M, Pryke C, et al. 2002 Nature 420 772; Scott P F, Carreira P, Cleary K, et al. 2003 Mon. Not. Roy. Astronom. S. 3411076

[4] Jarosik N, Barnes C, Greason M R, et al. 2006 Astrophys. J. submitted preprint astro-ph/0603452; Hinshaw G., Nolta M R, Bennett C L, et al. 2006 Astrophys. J. submitted preprint astroph/0603451; Page L, Hinshaw G, Komatsu E, et al. 2006 Astrophys. J. submitted preprint astro-ph/0603450

[5] Spergel D N, Bean R, Dorè et al. 2006 Astrophys. J. submitted preprint astro-ph/0603449

[6] Caldwell R R, Dave R and Steinhardt P J 1998 Phys. Rev. Lett. 80 1582; Wetterich C 1988 Nucl. Phys. B302 668

[7] Brax P and Martin J 1999 Phys. Lett. B468 40; Brax P and Martin J 2000 Phys. Rev. D61 103502

[8] Caldwell R R 2002 Phys. Lett. B545 23-29

[9] Armendariz-Picon C, Mukhanov V and Steinhardt P J 2000 Phys. Rev. Lett. 85 4438-4441; Armendariz-Picon C, Mukhanov V and Steinhardt P J 2001 Phys. Rev. D63 103510

[10] Feng B, Wang X, Zhang X 2005 Phys. Lett. B 607 35-41

[11] Carroll S M, Duvvuri V, Trodden M, et al. 2004 Phys. Rev. D70 043528; Nojiri S and Odintsov S D 2003 Phys. Rev. D68 123512

[12] Zlatev I, Wang L and Steinhardt P J 1999 Phys. Rev. Lett. 82 896-899; Steinhardt P J, Wang L and Zlatev I 1999 Phys. Rev. D59 123504

[13] Wang Y and Mukherjee P 2006 Astrophys. J. in press Preprint astro-ph/0604051

[14] Zhao G-B, Xia J-Q, Feng B and Zhang X 2006 Preprint astro-ph/0603621

[15] Jassal H K, Bagla J S and Padmanabhan T 2005 Mon. Not. Roy. Astronom. S. 356 L11-L16; Jassal H K, Bagla J S and Padmanabhan T 2005 Phys. Rev. D 72103503

[16] Ratra B and Peebles P J E 1988 Phys. Rev. D37 3406

[17] Weller J and Lewis A M 2003 Mon. Not. Roy. Astronom. S. 342 L72

[18] Amendola L and Quercellini C 2003 Phys. Rev. D 68 023514; Caldwell R \& Doran M 2004 Phys. Rev. D 69103517

[19] Barro-Calvo G and Maroto A L Preprint astro-ph/0604409

[20] Efstathiou G and Bond J R 1999 Mon. Not. Roy. Astronom. S. 304 75; Kosowsky A, Milosavljevic M and Jimenez R 2002 Phys. Rev. D66 063007

[21] Cole S., Percival W J, Peacock J A, et al. 2005 Mon. Not. Roy. Astronom. S. 362 505-534

[22] Astier P, Guy J, Regnault N, et al. 2006 Astronom.Astrophys. 447 31-48

[23] Klypin A, Macciò A V, Mainini R and Bonometto S A 2005 Astrophys. J. 599 31-37

[24] Alabidi L and Lyth D H 2006 Preprint astro-ph/0603539

[25] Xia J-Q, Zhao G-B, Feng B and Zhang X 2006 JCAP submitted Preprint astro-ph/0603393

[26] Mainini R, Colombo L P L and Bonometto S A 2005 Astrophys. J. 632 691-705

[27] Bludman S2004 Phys. Rev. D69 122002

[28] Kneller J P and Strigari L E 2003 Phys. Rev. D68 083517 